\title{
La Didáctica: Epistemología y Definición en la Facultad de Ciencias Administrativas y Económicas de la Universidad Técnica del Norte del Ecuador
}

\author{
Omar Abreu, Mónica C. Gallegos, José G. Jácome y Rosalba J. Martínez \\ Universidad Técnica del Norte, Campus. Avenida 17 de julio, 5-21, General José María Córdova, CP \\ 199, Ibarra, Ecuador. (e-mail: oabreu@utn.edu.ec, mgallegos@utn.edu.ec, jcuillermo@utn.edu.ec ${ }_{2}$ \\ rjmartinez@utn.edu.ec)
}

Recibido Oct. 28, 2016; Aceptado Ene. 4, 2017; Versión final Ene. 26, 2017, Publicado Jun. 2017

\begin{abstract}
Resumen
Se presenta un modelo didáctico integrador, para transformar el modo de actuación profesional relacionado con la didáctica de los docentes vinculados a la Facultad de Ciencias Administrativas y Económicas de la Universidad Técnica del Norte del Ecuador. La composición de la facultad es muy diversa y heterogénea y los académicos no cuentan con la experiencia y la preparación suficientes para responder a las demandas de la docencia universitaria de estos tiempos. Este estudio muestra los resultados de un estudio histórico y epistemológico de la didáctica, cuyo centro de atención es la evolución de su definición, resaltando limitaciones y aciertos. Se propone una nueva definición de este concepto con una visión más integral e integradora que incluye al docente y al estudiante.
\end{abstract}

Palabras clave: epistemología; rol docente; didáctica; enseñanza universitaria

\section{Didactics: Epistemology and Definition in the Faculty of Management and Economics at the Technical University of Northern Ecuador}

\begin{abstract}
An integrating didactic model aimed to transform the professional action related to the didactics of the professors of the Faculty of Administrative and Economic Sciences of the Technical University of North of Ecuador. The composition of the faculty members is very diverse and heterogeneous and professors do not have the experience and sufficient preparation to respond to the demands of modern university teaching. This study shows the results of a historical and epistemological study of didactics, whose center of attention is the evolution of its definition, highlighting limitations and success. A new definition of this concept that includes a more integral and integrative vision that includes teachers and students is proposed.
\end{abstract}

Keywords: epistemology; teacher role; didactic; university teaching 


\section{INTRODUCCIÓN}

En el mundo educacional una de las polémicas más antiguas, cuya vigencia parece eterna, por su contemporaneidad, gira alrededor de la Didáctica. El debate se ha centrado en sus orígenes, su condición de ciencia, su objeto de estudio, sus categorías, leyes, principios, modelos, evolución, su carácter general o disciplinar y en su relación con la Pedagogía y con las metodologías. La Didáctica es una respuesta a la necesidad de encontrar un equilibrio que armonice la relación entre las maneras de enseñar de los educadores y el aprendizaje de sus discípulos; una contradicción todavía por resolver. Varios autores se han referido al tema en cuestión, como Herbart (1935), Aebli (1958), Gottler (1962), Alves 81962), Stoker (1966), Buyse (1964), Titone (1970), Villalpando (1970) y Fernández (1970), Nérici (1973), Blankertz (1981), Pacios (1982), Pérez (1982), Fernández y Sarramona (1984), Benedito (1987), Rosales (1988), Contreras (1990), Sacristán (1989), Vasco (1990), Carvajal (1990), Camilloni (1994), Álvarez (1993), Álvarez (1997), Addine et al., (1998), Díaz (1999), Fuentes (2000), Gervilla (2000), Díaz (2001), Mallart (2001), Addine., et, al, (2004), Sevillano (2005), Hashimoto (2006), Zabalza (2007), Medina et al., (2009), Madrid y Mayorga (2010), Pla et al., (2010), Moreno (2011y Abreu et al., ( 2016) entre otros.

En la Facultad de Ciencias Administrativas y Económicas (FACAE), de la Universidad Técnica del Norte de Ecuador, se desarrolla un proyecto investigación cuyo objetivo es crear un modelo didáctico integrador para que sus docentes conduzcan exitosamente el aprendizaje desarrollador de los estudiantes. El diagnóstico aplicado por los investigadores reveló que el $27.8 \%$ de los docentes de la FACAE no reconoce que la Didáctica es una ciencia, el $8.8 \%$ plantea que es parte de la Pedagogía, el $15.2 \%$ dice que no tiene un objeto de estudio y el $13.9 \%$ no identifica su objeto de estudio. El $21.4 \%$ reconoció que dominar el contenido de la Didáctica no era su prioridad y que no tenía una importancia alta para sus clases. El $35.2 \%$ no estableció relación entre la Didáctica y el contenido de sus asignaturas en sus clases y para el $31.1 \%$ la Didáctica formaba parte de su modo de actuación profesional a veces o nunca.

El proyecto de investigación Modelo Didáctico Integrador para la Facultad de Ciencias Administrativas y Económicas de la universidad Técnica del Norte reveló que esta situación se relaciona con que el 53.8\% no había recibido cursos de formación didáctica, el 37.5\% no había estudiado Pedagogía, el $65 \%$ no se había vinculado con la metodología de las asignaturas que imparte y el $52.8 \%$ tiene menos de cinco años de experiencia en la Educación Superior. Esta realidad impidió el desarrollo de la capacidad necesaria para discernir entre la diversidad de criterios y posiciones teóricas en torno a la Didáctica y para resolver la problemática vigente alrededor de su valoración como arte, disciplina, asignatura, tecnología, saber técnico, teoría, conjunto de técnicas, campo del conocimiento, normativa, doctrina, metodología, rama o parte de la Pedagogía, como subconjunto o Pedagogía sistémica, ciencia y hasta su propia existencia o no.

Otra causa importante ha sido reconocida en la falta de vigor de la Didáctica como ciencia, su frecuente descontextualización, sus carencias investigativas y en la inconsistencia de su ejercitación, afectada por la rutina, el dogmatismo, la superficialidad, los desaciertos, la improvisación y el desatino. Esta situación relacionada con la ausencia de una Didáctica vigorosa ha sido percibida y denunciada permanente por sus destinatarios, los estudiantes (Díaz, 1999). El equipo de investigadores se propuso realizar un estudio epistemológico de la Didáctica y una redefinición de la misma que contribuyera a solucionar estas contradicciones, a la unificación de criterios y a la elevación del dominio del contenido de la Didáctica entre los profesores de la FACAE y su incorporación al modo de actuación profesional de estos.

A la Didáctica se le incluye entre las emergentes ciencias de la educación, cuya juventud a veces determina la inconsistencia y la diversidad de criterios en relación con su teoría. Los estudiosos de este complejo tema han encontrado consenso en el reconocimiento del teólogo, filósofo y pedagogo de origen checo Juan Amos Comenio (Uherský Brod, Moravia, 28 de marzo de 1592 - Ámsterdam,15 de noviembre de 1670), como el padre de esta criatura. Comenio publicó en 1640 "La Didáctica Magna" y la divido en tres partes: Didáctica General, Didáctica Especial y Organización Escolar. En esta obra reclamó la urgencia de buscar métodos de enseñar que alcanzaran la perfección. Comenio estableció tres principios básicos para el desarrollo didáctico: a) la Didáctica es una técnica y un arte, b) la enseñanza debe tener como objetivo el aprendizaje de todo por parte de todos, c) los procesos de enseñanza y aprendizaje deben caracterizarse por la rapidez y la eficacia, así como por la importancia del lenguaje y de la imagen (Comenio, 1998).

Otra figura relevante en los inicios del quehacer didáctico fue el suizo Johann Heinrich Pestalozzi (Zúrich, 12 de enero de 1746 - Brugg, 17 de febrero de 1827), cuya celebridad obedece a la divulgación de un método de enseñanza que se sustentó en la intuición como paso previo para para la formación de las ideas claras, según el desarrollo mental de los niños. Este método determinó el surgimiento de varias escuelas con su sello en Europa. Sus postulados son el punto de partida de la inconformidad constante con el estado de cosas que ocurren alrededor del desempeño del docente y el estudiante en el aula, de la enseñanza representada en el primero y el aprendizaje conceptualizado en el segundo e integrado en la categoría 
proceso de enseñanza- aprendizaje. La típica contradicción entre lo viejo y lo nuevo, lo estático y lo dialéctico, lo tradicional y lo alternativo. Estos son los cimientos para la aparición de la escuela nueva o escuela activa, cuyos aciertos no han sido suficientes para vencer la resistencia de las reminiscencias de la escuela tradicional.

Las primeras aportaciones a la Didáctica no significaron la aparición de un sistema teórico acabado y consistente que coronara con éxito la satisfacción de quienes se relacionan con ella, todo lo contario, el debate, la polémica, la experiencia y las investigaciones son cotidianos y los aportes, los cambios y los descubrimientos también "Con el transcurso del tiempo, el ámbito de la Didáctica General ha sufrido profundas transformaciones, debido al surgimiento de nuevas formas de conocimiento en educación, a los importantes avances de la investigación educativa, a la aparición de teorías psicológicas del aprendizaje, a la forma de concebir la inteligencia humana, entre otras" (Moreno, 2011). En la revisión de la bibliografía desde los inicios hasta hoy, no existe un concepto único y una definición uniforme de la Didáctica (Estebaranz, 1999), pasando por la síntesis de sus muchas definiciones. Esta realidad determinó que entre los docentes de la FACAE se asumieran cinco posiciones relacionadas con la Didáctica.

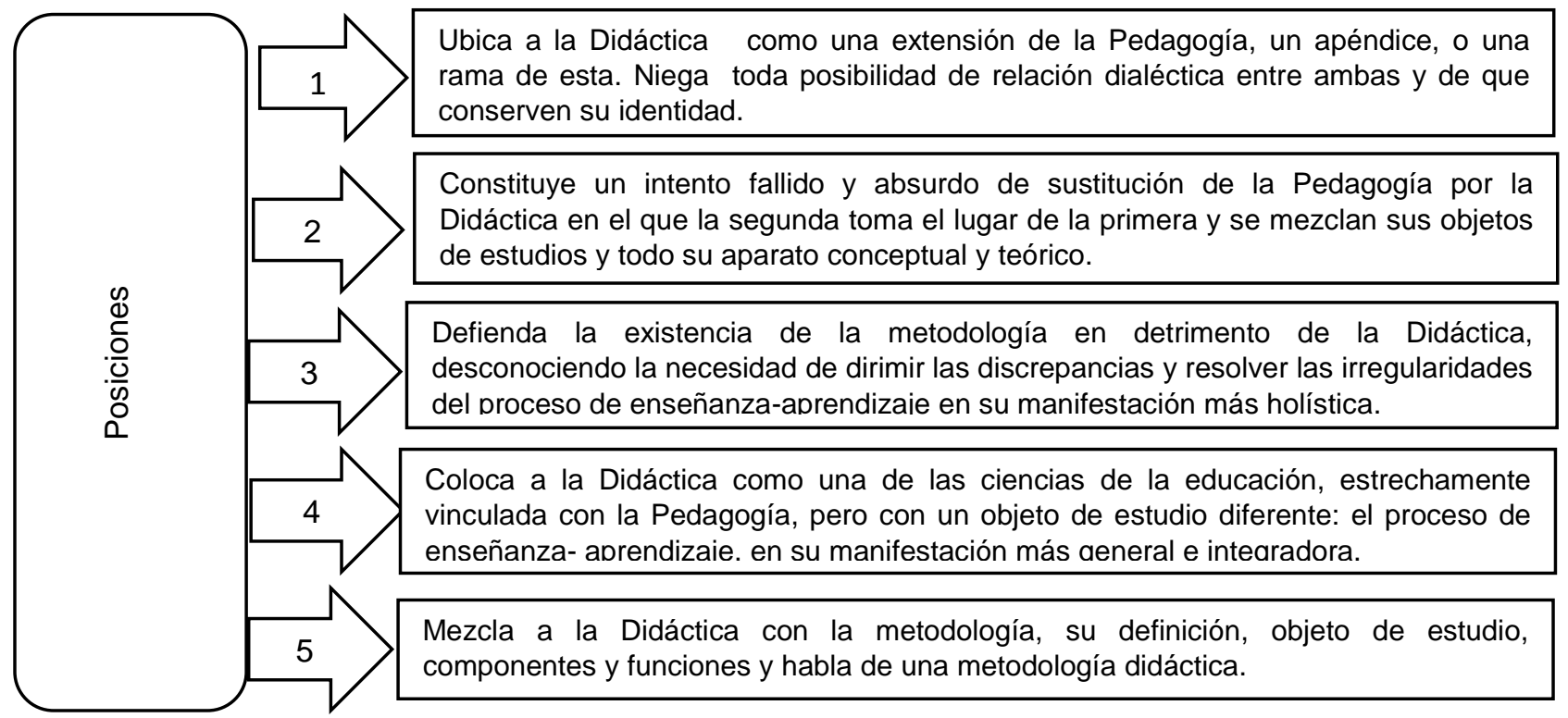

Fig. 1: Posiciones relacionadas con la Didáctica reveladas por los docentes de la FACAE en el diagnóstico

\section{METODOLOGÍA}

El equipo de investigadores hizo coincidir población y muestra considerando que en la Facultad laboran 127 docentes y que existen facilidades para trabajar con todos. Se utilizaron el método histórico lógico y analítico sintético para estudiar la historia de las definiciones de la Didáctica, el dialéctico para la epistemología, el inductivo y el genético para llegar a la nueva definición de la Didáctica. Para evaluar la calidad del trabajo se utilizó la metodología de la comparación por pares del criterio de expertos. Se seleccionaron 15 expertos, 5 ecuatorianos, 5 cubanos, 1 boliviano, 2 españoles, 1 argentino y 1 colombiano. El promedio de años de experiencia profesional es de 17 años. Entre los expertos, 6 son Doctores en Ciencias Pedagógicas, el resto en otras especialidades, pero todas vinculadas a la docencia universitaria. Para evaluarlos se les indicó que marcaran con una cruz (X), en una escala creciente de 1 a 10 , el valor que se corresponde con el grado de conocimiento e información acerca del tema objeto de investigación, considerando los indicadores siguientes: 1) participación en investigaciones relacionadas con el tema, 2) estudios teóricos relacionados con el tema, 3) participación en eventos internacionales relacionados con el tema y 5) nivel de conocimientos acerca de la Didáctica.

Tabla 1: Resultados de la autoevaluación de los expertos

\begin{tabular}{|l|l|l|l|l|l|l|l|l|l|}
\hline 1 & 2 & 3 & 4 & 5 & 6 & 7 & 8 & 9 & 10 \\
\hline 0 & 0 & 0 & 0 & 0 & 0 & 2 & 2 & 5 & 6 \\
\hline
\end{tabular}

A los expertos se les hizo llegar un cuestionario para evaluar con las categorías muy adecuado (MA), bastante adecuado (BA), adecuado (A), poco adecuado (PA) o no adecuado (NA) a los siguientes indicadores: 1) vigencia del objeto de estudio, 1) planteamiento del objeto de estudio, 3) método empleado, 4) resultados del estudio epistemológico y 5) nueva definición de la Didáctica. 
Como parte del proyecto de investigación se desarrolló un curso de capacitación a manera de taller concebido como espacios para el trabajo en equipo con las fuentes del contenido bajo la conducción del profesor para el análisis, la síntesis, la integración, la presentación y el debate de la información recopilada y procesada para ejecutar las tareas orientadas previamente. Los talleres propiciaron la profundización, la generalización, la integración y la aplicación del contenido a situaciones cognoscitivas y metacognoscitivas nuevas. En el taller integrador los docentes expusieron todo el contenido relacionado con el tema y lo vincularon con situaciones propias de su profesión. Demostraron los niveles de conocimientos y las habilidades desarrolladas en el PEA del tema, la aplicación de este contenido en su actuación como miembros activos de la comunidad en su gestión transformadora de la sociedad y el desarrollo de su capacidad comunicativa mediante la exposición de los resultados del proceso investigativo (Abreu, et. al. 2016). Se utilizó el método de elaboración conjunta mediante el desarrollo de situaciones problémicas que implicaron un estudio epistemológico de la Didáctica, concentrado en fuentes originales y no en el uso de fragmentos aislados y descontextualizado citados por otros autores y repetidos en la literatura especializada.

Tabla 2: Evaluación realizada por los expertos

\begin{tabular}{|c|l|l|l|l|l|l|c|}
\hline$N^{\circ}$ & \multicolumn{1}{|c|}{ Indicadores } & $M A$ & $B A$ & $A$ & $P A$ & $N A$ & Categoría definitiva \\
\hline 1 & Vigencia del objeto de estudio & 15 & & & & & MA \\
\hline 2 & Planteamiento del objeto de estudio & 15 & & & & & MA \\
\hline 3 & Método empleado & 9 & 3 & 3 & & & BA \\
\hline 4 & Resultados del estudio epistemológico & 13 & 2 & & & & MA \\
\hline 5 & Nueva definición de la Didáctica & 5 & 3 & 6 & 1 & & $\mathrm{~A}$ \\
\hline
\end{tabular}

\section{RESULTADOS Y DISCUSIÓN}

El estudio generó tres resultados: un estudio epistemológico de la Didáctica, sustentado en el análisis de las fuentes originarias, una definición más abarcadora y contextualizada de la Didáctica y la transformación del nivel de conocimientos didácticos de los docentes de la FACAE, los cuales se describen a continuación.

\section{La Didáctica antes de los años ochenta del siglo XX}

La Didáctica ha sido definida indistintamente como arte de enseñar, artificio, tratado, normativa, aprendizaje estudio científico, estudio de la educación intelectual del hombre y del conocimiento sistemático, ciencia auxiliar, técnica de incentivar, teoría de la instrucción, ciencia especulativa, doctrina general, método, técnica, procedimiento, disciplina particular, rama de la Pedagogía, disciplina pedagógica, disciplina pedagógica de carácter práctico normativo, disciplina reflexivo aplicativa, conjunto de técnicas de enseñanza, teoría de la instrucción y de la enseñanza, teoría práctica, teoría general de la enseñanza, metodología de instrucción, metodología que estudia los métodos y procedimientos en las tareas de la enseñanza y del aprendizaje, conjunto de métodos, ciencia especulativa y tecnológica, conjunto de interacciones, reflexión científica, campo de conocimiento, campo científico, tecnología, ciencia y tecnología, ciencia y técnica, ciencia de enseñar y aprender y ciencia. La mayoría de la autores elaboran sus definiciones de la Didáctica concentrando la atención en el objeto de estudio de la esta ciencia, lo que constituye una manera simple y poco convincente, que mutila el verdadero alcance de esta ciencia y limita los estudios epistemológicos a repetir lo que han dicho otros sin asumir una posición crítica.

La lógica histórica aconseja que el análisis epistemológico de la Didáctica se realice al menos en dos momentos, antes de la década del ochenta del siglo XX y después de este período. La década de los ochenta del siglo XX tuvo un peso fundamental en el estallido del interés, las investigaciones y las aportaciones a la Didáctica, por la convergencia de factores, que condicionaron su replanteamiento y reconceptualización (Roselló, 2005). La literatura disponible ofrece un variado y numeroso volumen de definiciones de la Didáctica. En la edición Nro 23 de 2014 de El diccionario de la Lengua Española, obra lexicográfica de la Academia, se conceptualiza a la Didáctica como "Perteneciente o relativo a la Didáctica o la enseñanza. Propio, adecuado o con buenas condiciones para enseñar o instruir. Un método, un profesor muy didáctico. Que tiene como finalidad fundamental enseñar o instruir. Género didáctico. Arte de enseñar".

Entre los pensadores griegos, cuna de la civilización humana y de las nuevas maneras de comprender el mundo, aparecieron los primeros acercamientos etimológicos a la Didáctica, en todos los casos con alusiones, imprecisas y generales, pero útiles, a la enseñanza y al aprendizaje. La palabra didáctica tiene su origen en el término diaktiqué, asociado al arte de enseñar. "En su etimología griega, la idea de Didáctica estuvo vinculada a muy diversos significados: la didáctica como el acto de enseñar; el didacta como 
instructor cualificado para enseñar; los manuales y métodos de enseñanza como recursos didácticos; las escuelas como instituciones especializadas en la didáctica; el proceso de aprendizaje como actividad central del aprendiz y propósito esencial de la actuación didáctica" (Zabalza, 2007). El término Didáctica procede del griego: didaktiké, didaskein, didaskalia, didaktikos, didasko (didaktike, didaskein, didaskalia, didaktikos, didasko). Todos estos términos tienen en común su relación con los verbos enseñar, instruir, exponer con claridad. Didaskaleion era la escuela en griego; didaskalia, un conjunto de informes sobre concursos trágicos y cómicos; didaskalos, el que enseña; y didaskalikos, el adjetivo que se aplicaba a la prosa didáctica.

La palabra didáctica fue empleada por primera vez relacionada con el sentido y la necesidad de enseñar en 1929, por el alemán Wolfgang Ratked en su libro Aphorisma Didactici Precipui o sea Principales Aforismos Didácticos (Sánchez 2012), pero fue desarrollada por Juan Amós Comenio quien también realizó su aporte en la búsqueda interminable por encontrar una definición que haga justicia a las verdaderas dimensiones y el significado real de la Didáctica. La definió como "el artificio fundamental para enseñar todo a todos. Enseñar realmente de un modo cierto, de tal modo, que no pueda no obtenerse un buen resultado. Enseñar rápidamente, sin molestias ni tedio ni para el que enseña ni para el que aprende, antes al contrario, con gran atractivo y agrado para ambos. Y enseñar con solidez, no superficialmente, no con meras palabras, sino encaminando al discípulo a las verdaderas, a las suaves costumbres" (Comenio, 1998). La definición ofrecida por Comenio, mirada a luz del tiempo histórico en que vivió, es aportativa y de mucho valor como punto de apartida de todo análisis de la Didáctica. Asoció a la Didáctica con el arte, sinónimo de exquisitez y de sensibilidad y creatividad. La definió como un fundamento esencial para el desarrollo de la enseñanza y la obtención de resultados positivos, buenos, provechosos y útiles, como un recurso para vencer el tedio, el aburrimiento y la monotonía, factores aún vigentes en la docencia contemporánea, y para hacer de la clase un acto entretenido, movilizador de la atención de los estudiantes y catalizador del interés. Complementa su punto de vista con la inclusión acertada del aprendizaje y lo condiciona al desarrollo de la enseñanza con las características mencionadas y sustentada en lo objetivo, en lo práctico, en la verdad, para que sea duradero, no efímero, temporal o superficial, sino sólido, profundo y convincente.

Herbart (1935) se refirió a la Didáctica como el resultado del estudio científico o de la combinación entre la enseñanza y la instrucción y consideró a la primera como la vía para lograr lo instructivo y a la última como medio de concreción de la educación. Aebli (1958) escribió que la Didáctica es una ciencia auxiliar y aplicada de la Pedagogía para la realización de tareas educativas que tienen como finalidad deducir el conocimiento psicológico de los procesos de formación intelectual y revelar las técnicas metodológicas más adecuadas para el desarrollo de tal proceso. Desde este punto de vista no se establecen límites entre Pedagogía, Didáctica y metodología; son lo mismo. Gottler (1962) refirió que la Didáctica es una teoría cuyo centro de atención es la instrucción educativa. La conceptualiza como ciencia, pero con un carácter especulativo. Alves (1962) considera que la Didáctica es la disciplina pedagógica de carácter práctico y normativo, el conjunto sistémico de principios, normas, recursos y procedimientos específicos que sirven para aprender los contenidos en estrecha vinculación con los objetivos educativos propuestos. Buyse (1964) dice que la Didáctica es la rama de la Pedagogía encargada de establecer las regulaciones para el desarrollo de la práctica docente. Stoker (1964) hizo público su criterio en el que refiere que la Didáctica es una teoría que sustenta el proceso de instrucción y enseñanza en toda la amplitud y totalidad de los niveles escolares. Concretamente dice: "La didáctica general plantea las cuestiones generales de toda la enseñanza comunes a todas las materias, intenta exponer los principios o postulados que en todas las asignaturas se presentan y que ha de ser objeto de consideraciones fundamentales" y Tomachewski (1967) asume una posición en la que manifiesta que la Didáctica es la teoría general de la enseñanza y que se centra por tanto en el conjunto de principios y técnicas que tiene un carácter general en tanto son aplicables a todas las disciplinas de este proceso.

Titone (1970) en su Metodología didáctica, polémico desde la definición del título de su publicación, asume la Didáctica en dos sentidos. En el primero, concibe a la Didáctica como como una metodología para la instrucción y en el segundo, como una tecnología de enseñanza. Villalpando (1970) describe a la Didáctica como la parte de la Pedagogía que establece los procederes para conducir al educando en un proceso progresivo de adquisición de conocimientos que incluye técnicas y hábitos, la organización más conveniente del contenido que debe asimilar el estudiante y los elementos que auxilian al docente en su conducción.

En los años que anteceden a la década de los ochenta del siglo XX la Didáctica no había logrado llamar la atención de un grupo numeroso de investigadores, ni de estudiosos con el poder movilizativo de los años posteriores. La explosión de intereses, teóricos y prácticos acerca de la Didáctica, cuyas definiciones fueron diversas, lejos de aminorarse se ha acrecentado y la polémica en torno a la teoría que la sustenta y los argumentos que se utilizan en la defensa de cada posición son tan variados como cuestionables en múltiples ocasiones. 
En todas las posiciones, no obstante la heterogeneidad de términos, se aprecia un consenso relacionado con que la Didáctica busca un equilibrio entre la manera de enseñar y el resultado del aprendizaje, pero ninguna resuelve convincente la interrogante, devenida problema, ¿qué es la Didáctica? Fernández (1970) "A la didáctica general le corresponde el conjunto de conocimientos didácticos aplicables a todo sujeto, mientras la didáctica especial es todo el trabajo docente y métodos aplicados a cada una de las disciplinas o artes humanas dignas de consideración. La didáctica especial tiene un campo más restringido que la didáctica general, por cuanto se limita a aplicar las normas de ésta, al sector específico de la disciplina sobre la que versa". Su definición establece diferencias entre dos Didácticas, una general y otra especial, pero tampoco logra acercarse a la solución del problema que significa ofrecer una definición objetiva y práctica de la Didáctica, que constituya un instrumento orientador para los docentes.

Nérici (1973) constituye una de las primeras referencias que asocia a la Didáctica con la ciencia, con la enseñanza y el aprendizaje. Establece una dialéctica entre conocimiento de la Didáctica, eficiencia de la enseñanza e intereses y características individuales y sociedad en general "(...) el estudio de la didáctica es necesario para que la enseñanza sea más eficiente, más ajustada a la naturaleza y a las posibilidades del educando y de la sociedad, es el conjunto de técnicas destinado a dirigir la enseñanza mediante principios y procedimientos aplicables a todas las disciplinas, para que el aprendizaje de las mismas se lleve a cabo con mayor eficiencia". Opina que la Didáctica posee capacidad para la integración de saberes, fruto de la actividad práctica y creadora de los docentes que derivan y perfeccionan el conjunto de técnicas que se aplican para el desarrollo de la enseñanza. Concreta que "La didáctica es un conjunto de técnicas a través de las cuales se realiza la enseñanza; para ello reúne con sentido práctico todas las conclusiones que llegan a la ciencia de la educación". Finalmente ofrece una definición más acabada y práctica y considera que la Didáctica es (...) el estudio del conjunto de recursos técnicos que tienen por finalidad dirigir el aprendizaje del alumno, con el objeto de llevarle a alcanzar un estado de madurez que le permita encarar la realidad, de manera consciente, eficiente y responsable, para actuar en ella como ciudadano participante y responsable".

\section{ESTUDIO EPISTEMOLÓGICO}

Esta sección se presenta en tres subsecciones para mayor claridad: (i) La Didáctica después de los años ochenta del siglo XX; (ii) La Didáctica: su nueva definición, y iii) La transformación del nivel de conocimiento didáctico de los docentes de la FACAE

\section{La Didáctica después de los años ochenta del siglo XX}

En la década del ochenta del siglo XX hubo un estallido de intereses investigativos, teóricos y prácticos acerca de la Didáctica que trasciende hasta nuestros días, que involucró a varias áreas geográficas, pero que tuvo una expresión relevante dentro del continente latinoamericano. La curiosidad que sintieron muchos investigadores de las ciencias sociales, especialmente los dedicados a las ciencias de la educación y particularmente al ejercicio de la docencia, hizo que la atracción investigativa por esta materia alcanzara dimensiones nunca vistas en este nuevo marco histórico. La avalancha de incursiones en torno a la teoría de la Didáctica no significó el fin de la polémica centrada en su cuerpo teórico, todo lo contario, avivó más el contrapunteo y ensanchó el espectro de posibilidades reales y subjetivas para la emisión de criterios, muchas veces dispersos, imprecisos y contradictorios, que han mantenido la vigencia del debate hasta la realidad educativa contemporánea, desde cuya perspectiva no se avizora el horizonte del diálogo final.

Llegar al punto convergente que acerque a los académicos contemporáneos a una definición consensuada acerca de la Didáctica parece todavía una quimera, aunque la utopía es más realizable en la segunda decena del siglo XXI "Innegablemente, la Didáctica ha experimentado, igual que las demás Ciencias Sociales, las vicisitudes de las indefiniciones epistemológicas, conceptuales y metodológicas" (Díaz, 2001). La cuestión no responde solo a la desenfrenada vocación por polemizar; se trata de otros factores "En la actualidad, enseñar se hace cada vez más complejo y aprender se ha convertido en una experiencia mucho más desafiante para los alumnos" (Moreno, 2011). El creciente interés por la Didáctica como ciencia no es casual. Está vinculado con la complejidad del mundo, con la necesidad de formación del talento humano para garantizar el desarrollo, con la aparición de nuevos problemas y conflictos y la persistencia de otros, con el insistente reclamo del derecho a la educación y la proximidad a la sociedad del conocimiento.

Tras la caída de muro de Berlín, el fin de la "Guerra Fría" y la expiración del conflicto Este- Oeste, se celebró en 1990, la Conferencia Mundial de Educación para Todos, auspiciada por la Organización de las Naciones Unidas para la Educación, la Ciencia y la Cultura (UNESCO), el Programa de las Naciones Unidas para el Desarrollo (PNUD), el Fondo Internacional de Emergencia de las Naciones Unidas para la Infancia (UNICEF) y el Banco Mundial. Allí surgió "El movimiento de Educación para Todos", un compromiso mundial de garantizar educación básica de calidad a todos los niños, jóvenes y adultos, una visión ampliada 
del aprendizaje. En el cónclave se formularon ocho objetivos, el segundo de ellos fue lograr la enseñanza primaria universal: velar por que todos los niños y niñas puedan terminar un ciclo completo de enseñanza primaria. En el año 2000 en Dakar, Senegal, se ratificó el compromiso relacionado con la Educación para Todos y el plazo se entendió hasta 2015. Para lograr el objetivo de 1990, se definió: ampliar el cuidado y la educación de la primera infancia, proporcionar educación primaria gratuita y obligatoria para todos, promover el aprendizaje y las habilidades para la vida de los jóvenes y adultos, aumentar la alfabetización de adultos, conquistar la igualdad de género y mejorar la calidad de la educación.

Tabla 3: Factores que contribuyeron al desarrollo de la Didáctica en década del ochenta del siglo XX

\begin{tabular}{|l|l|l|}
\hline Contextos & \multicolumn{1}{|c|}{ Factores } & \multicolumn{1}{c|}{ Manifestaciones } \\
\hline $\begin{array}{l}\text { Científico y } \\
\text { epistemológico }\end{array}$ & $\begin{array}{l}\text { Aparecen y se desarrollan nuevas } \\
\text { formas de entender y construir el } \\
\text { conocimiento científico, fruto de la la } \\
\text { experiencia práctica y de } \\
\text { investigación científica. }\end{array}$ & $\begin{array}{l}\text { Un cuestionamiento al positivismo como paradigma lineal y } \\
\text { objetivista y único método del conocimiento científico. } \\
\text { Aparecen nuevas formas metodológicas para la obtención del } \\
\text { conocimiento científico, cuyo centro es la esencia de los } \\
\text { problemas que los generan. La interpretación y la crítica } \\
\text { alcanzan un protagonismo importante. }\end{array}$ \\
\hline Social & $\begin{array}{l}\text { La incursión en la postmoder- } \\
\text { nidad es más frecuente e intensa } \\
\text { y el desarrollo de la sociedad del } \\
\text { conocimiento más acelerado, } \\
\text { exigente y necesario. }\end{array}$ & $\begin{array}{l}\text { Las instituciones educativas enfrentas nuevas exigencias, } \\
\text { sobre la base del surgimiento de otras formas de } \\
\text { pensamiento, de percibir e interpretar la realidad, de } \\
\text { relaciones humanas, de enseñar y aprender y de actuar en la } \\
\text { práctica. }\end{array}$ \\
\hline Profesional & $\begin{array}{l}\text { Cambio de roles } \\
\text { Se introducen nuevas formas organizativas en las instituciones } \\
\text { educativas y aparecen otros contextos para la formación, lo } \\
\text { que genera cambios en el rol profesional del docente en } \\
\text { concordancia con el nuevo contexto. }\end{array}$ \\
\hline
\end{tabular}

El objetivo propuesto no se logró. El 25 de diciembre de 2015, la Asamblea General de Naciones Unidas, aprobó una en la que estableció 17 objetivos para el 2030, relacionados con problemas del mundo. Uno de ellos consiste en garantizar una educación inclusiva y equitativa de calidad y promover oportunidades de aprendizaje permanente para todos. Esta realidad determinó que las inversiones en la educación aumentaran, en una mezcla de nobles aspiraciones y ambiciones económicas. Las inversiones mundiales en educación ascienden a 2000 billones de dólares, más del doble del mercado mundial del automóvil. Es por esto que es un área atractiva y de gran potencial para un capital ávido de nuevas áreas de valorización. Desde el inicio de la década de 1990, los analistas financieros han llamado la atención para el potencial que tiene la educación para transformarse en uno de los más vibrantes mercados del siglo XXI (Sousa, 2007). Esta realidad también contribuyó a nuclear la atención y el interés alrededor de la Didáctica.

Blankertz (1981), quien había afirmado que la pedagogía del nazismo tenía su raíz en el culto a la personalidad del Hitler entre la juventud burguesa alemana, planteó con cierta inseguridad, que parecía adecuado decir que la Didáctica y lo didáctico se refieren (como sustantivo y adjetivo) a actividades de enseñanza o de formación en general. Pacios (1982) asegura que la Didáctica es una ciencia especulativa y tecnológica. Pérez (1982) la considera como ciencia y tecnología del sistema de comunicación intencional, donde se desarrollan los procesos de enseñanza-aprendizaje, orientada a optimizar la formación intelectual.

Fernández y Sarramona (1984) dicen que es la ciencia de la educación de carácter teórico-normativo que busca la adquisición de hábitos intelectuales mediante la integración del aprendizaje de los bienes culturales. Benedito (1987) cree que la Didáctica está en camino de ser ciencia y tecnología a la vez, pero aún no lo es, que se construye, desde la teoría y la práctica, en ambientes organizados de relación y comunicación intencional, donde se desarrollan procesos de enseñanza y aprendizaje para la formación del alumno. Rosales (1988) alega que es la ciencia del proceso de enseñanza sistemática, en cuanto optimizadora del aprendizaje y Contreras (1990) la ve como la disciplina que explica los procesos de enseñanza-aprendizaje para proponer su realización, consecuente con las finalidades educativas. En un lapsus de tiempo que no supera la decena de años estos autores consideran que la Didáctica es disciplina, ciencia en formación, ciencia complementaria y ciencia indistintamente y recurren al objeto de estudio como elemento determinante en su definición.

Sacristán (1989) ofreció un nuevo punto de vista "La Didáctica, como disciplina científica a la que corresponde el guiar a la enseñanza, tiene un componente normativo y otro prescriptivo (...) es ciencia, arte y praxis". El primero de los componentes se encarga de establecer las normas bajo las cuales se desarrolla la enseñanza y el segundo de los estándares terminológicos necesarios, según el autor, para la comprensión y aplicación de la teoría didáctica. Vasco (1990) revela un criterio desde cuya óptica 
considera a "la Didáctica no como la práctica misma del enseñar, sino como el sector más o menos bien limitado del saber pedagógico que se ocupa explícitamente de la enseñanza". Carvajal (1990) escribió que la Didáctica "Es la ciencia de la educación que estudia e interviene en el proceso de enseñanzaaprendizaje con el fin de conseguir la formación intelectual del educando, (...) es parte de la pedagogía que se interesa por el saber, se dedica a la formación dentro de un contexto determinado por medio de la adquisición de conocimientos teóricos y prácticos, contribuye al proceso de enseñanza aprendizaje, a través del desarrollo de instrumentos teóricos-prácticos, que sirvan para la investigación, formación y desarrollo integral del estudiante".

De la Torre (1993) definió a la Didáctica como una disciplina pedagógica cuyo centro gira alrededor de los procesos de formación en contextos deliberadamente organizados. Álvarez (1993) no duda en reconocer la condición de ciencia de la Didáctica y enmarca su evolución dentro de la búsqueda de las soluciones de los problemas contemplados en el proceso docente-educativo de las instituciones educacionales, cuya función es preparar al ser humano para los retos de la vida de manera sistémica y eficiente. Para este autor la Didáctica "(...) es una ciencia social, humanística y sus leyes tienen una naturaleza dialéctica. Cervantes (1996) plantea que la Didáctica es un aspecto de manifestación práctica inherente a la Pedagogía, que tiene como sustento una teoría científica.

Camilloni (1994) desde su posición teórica asegura que la Didáctica es la teoría de la enseñanza, heredera y deudora de muchas otras disciplinas, que al ocuparse de la enseñanza se constituye en oferente y dadora de teorías en el campo de la acción social y del conocimiento. En estas tres definiciones salta a la vista la identificación de la Didáctica con uno de los componentes esenciales de su núcleo sistémico; la enseñanza. El aprendizaje no entra en esta cobertura, ni siquiera como un proceso diferenciado, mucho menos como manifestación de un proceso único. Para Álvarez (1997), también la Didáctica es una ciencia, pero no acabada, en construcción, que se nutre de la realidad educativa sometida, a su vez, al análisis y a la reflexión de educadores y educandos. La autora defiende en su propuesta la relación estrecha de la Didáctica y la Pedagogía y asegura que se alimenta de la reflexión colectiva, del análisis y la observación de la compleja realidad educativa y para su desarrollo aplica métodos y técnicas, propios de la investigación que la enriquecen y contribuyen a su crecimiento teóricopráctico.

Otros investigadores aseveran que "En los últimos años la Didáctica se ha revalorizado, lo que está asociado al avance en la elaboración de un cuerpo teórico propio. La Didáctica actual es ese campo de conocimientos, de investigaciones, de propuestas teóricas y prácticas que se centran, sobre todo, en los procesos de enseñanza y aprendizaje" (Addine et al., 1998). Díaz (1999) explica que la Didáctica pude definirse como la ciencia que explica y aplica lo relacionado con la enseñanza como la tarea intencional y sistemática y como la estrategia expedita para lograr la formación del hombre. Fuentes (2000) dice que es una ciencia asociada al proceso de formación profesional y que se expresa en funciones, contradicciones, categorías y leyes y que existen la Didáctica General y las Didácticas Especiales. La primera posibilita la concepción, estructuración y el desarrollo de dicho proceso en sus aspectos más generales y la segunda desempaña la misma función en un marco más estrecho; en las ciencias, las tecnologías y las artes particulares. También insiste en la idea de la existencia de la Didáctica de la Educación Superior, de la cual depende la efectividad, eficacia y la eficiencia de los profesionales encargados del desarrollo integral de cada país.

Gervilla (2000) la concibe como la ciencia de la educación que estudia todo lo relacionado con la enseñanza: diseño de las mejores condiciones, ambiente, clima, para conseguir un aprendizaje excepcional y el desarrollo completo del alumno. Díaz (2001) plantea la relación de la Didáctica con otras disciplinas del saber académico, pero siempre asociada a la enseñanza y escribe que "puede definirse como un marco explicativo interdisciplinario donde confluyen los aspectos filosóficos, psicológicos y sociológicos de la enseñanza, los cuales permiten dar profesionalismo y cientificidad al acto educativo en cualquier nivel y en cualquiera asignatura. Mallart (2001) también se adhiere a la definición de la Didáctica como la ciencia de la educación, cuya finalidad consiste en lograr la formación intelectual de los educandos.

Sevillano (2004) la considera como la ciencia teórico-normativa que guía intencionalmente el proceso optimizador de enseñanza-aprendizaje, en un contexto determinado e interactivo y posibilita la aprehensión de la cultura con el fin de conseguir el desarrollo integral del estudiante. Otros estudiosos reconocen los avances de la Didáctica como ciencia en los últimos 20 años, como consecuencias de la reflexión, el debate y los aportes de los participantes en el proceso de enseñanza-aprendizaje, complejo y multifactorial. Plantean que la Didáctica es una teoría práctica, cuya realización principal consiste en el desarrollo de principios de contextualización e intercomunicación entre las teorías, con el fin de evitar el dogmatismo de carácter metodológico (Addine., et, al, 2004). El alcance de la Didáctica no se reduce a la enseñanza; su dimensión se complementa con la integración del aprendizaje y su comprensión como proceso. 
Hashimoto (2006) trae a colación uno de los elementos más discutidos de la vieja polémica. Categórica y abiertamente niega que la Didáctica es una ciencia, y alega que las definiciones de la Didáctica, su quehacer, el análisis lógico y epistemológico, conducen a una conclusión sin mucho margen de contradicción; la Didáctica no es una ciencia. Asegura que la Didáctica es una tecnología de la educación y como tal estructura, desarrolla, y transforma el proceso de enseñanza-aprendizaje con el propósito de viabilizar la formación eficiente, eficaz y con calidad del estudiante. Es tecnología, según el autor, porque utiliza la técnica en el proceso formativo conscientemente y basada en la ciencia y reflexiona acerca de ellas para mejorarlas y porque tiene un fin determinado.

Zabalza (2007) retoma el análisis de la enseñanza y el aprendizaje como proceso asociado a la Didáctica y la define como ese campo de conocimientos, de investigaciones, de propuestas teóricas y prácticas que se centran en los procesos de enseñanza y aprendizaje: cómo estudiarlos, cómo llevarlos a la práctica en buenas condiciones y cómo mejorar todo el proceso. Medina et al., (2009) describe a la Didáctica como la disciplina o tratado riguroso de estudio y fundamentación de la actividad de enseñanza en cuanto propicia el aprendizaje de los estudiantes en los más diversos contextos. Madrid y Mayorga (2010) consideran que la Didáctica es el campo de acción de numerosas investigaciones de proyecciones teóricas y prácticas, cuyos aportes han enriquecido el sistema de conocimientos y han determinado su carácter de ciencia en dos dimensiones, la teoría y la práctica.

Pla et al., (2010) establece una relación entre la Pedagogía y la Didáctica, manifestó que "La Didáctica es una rama de la Pedagogía, que adquiere el carácter de ciencia en la medida que estudia un nivel cualitativo de organización del proceso educativo que posee peculiaridades, que tienen que ver con las relaciones internas que se producen entre el educador y el alumno mediados por los componentes: objetivos, contenidos, métodos, formas, medios, evaluación desde un objeto preciso del conocimiento" y Moreno (2011) analizó los nuevos desafíos que tendría que enfrentar la Didáctica en el contexto del siglo XXI, en la época de la sociedad del conocimiento.

Desde estos horizontes. "La didáctica no debe concebirse únicamente como un variado conjunto de estrategias docentes amplias y coherentes con la finalidad de que el profesor pueda aplicarlas en el contexto del aula". Asumir tal posición sería simplificar, minimizar, reducir el alcance esta ciencia. La Didáctica debe ser abordada desde posiciones teóricas que pueden variar según los enfoques que se asumen para el ejercicio de la docencia. En 2016 investigadores de la Universidad Técnica del Norte, de la república del Ecuador, se adhiere a la posición que defiende la dialéctica entre la Pedagogía y la Didáctica, la condición de ciencia de ambas y sus particularidades, así como la vigencia del debate académico relacionado con la polémica del tema, pero deslindan la esencia de cada una y las diferencias que preservan la esencia de cada cual (Abreu et al., 2016).

\section{La Didáctica: su nueva definición}

Enunciar una definición es un reto, una tarea profesional de alta complejidad teórica. La definición es un procedimiento científico, un ejercicio de pensamiento, un esfuerzo y una construcción intelectual; una proposición lógica, precisa, objetiva, coherente, concreta, peculiar y orientadora relacionada con los objetos, los seres, los fenómenos, las cosas y las ideas, que propicia la representación gráfica de estos y la comprensión de los conceptos que los tipifican, que los revelan como tales, de la manera más inequívoca posible permitida por la profundidad del conocimiento que se posea acerca de ellos. Para lograr una definición de la Didáctica como ciencia que haga justicia a su función e importancia para el desarrollo de la educación, es necesario concebirla mental, teórica y prácticamente en toda su dimensión, en la totalidad de su alcance dentro de la multiplicidad de relaciones y sucesos que ocurren en el complejo proceso que se desarrolla dentro de cada aula de una institución educativa, en función de un aprendizaje verdaderamente desarrollador, asumido por el docente y por el estudiante como destinatario y principal beneficiario del mismo, cuyo colofón es la formación integral de la personalidad en un contexto diverso, cambiante, exigente, polémico, contradictorio en el que existe una comunidad de intereses diferentes, reconocibles, conciliables y respetables.

Como resultado del proyecto se elaboró una nueva definición de la Didáctica: es una de las ciencias de la educación en pleno desarrollo. Está estrechamente vinculada con otras ciencias que intervienen en el proceso de enseñanza aprendizaje integrado e institucionalizado, especialmente con la Pedagogía, pero conserva sus particularidades y su esencia propia. Como ciencia orienta, socializa, integra y sistematiza en un cuerpo teórico en evolución ascendente, continua y sistemática, los resultados investigativos y de la experiencia acumulada en la práctica educativa, orientados a la exploración de la realidad del aula, a la detección, el estudio y la búsqueda de soluciones acertadas de los problemas que afectan e impiden el desarrollo óptimo, eficaz y eficiente del proceso de enseñanza-aprendizaje en su manifestación más amplia y contemporánea, 
que implica emocional y físicamente a profesores y estudiantes y los coloca en posición de éxito, en roles diferentes, pero con un propósito similar, a los primeros como guías, conductores del mismo y a los últimos como sujetos de su propio aprendizaje capaces de aprender el contenido de las asignaturas y los métodos para conseguirlo y de valorar críticamente las estrategias aplicadas para lograrlo, revela al método como parte del contenido, crea y desarrolla estructuras de participación que se sustentan en el diálogo y la retroalimentación, que facilitan la construcción y el desarrollo del aprendizaje, concebido, ejecutado y dirigido en el marco de instituciones educativas, para explicar, relacionar, demostrar y aplicar conocimientos necesarios para la vida práctica, en función de la formación integral de la personalidad, mediante el ascenso progresivo de la dependencia a la independencia autorregulada y a la capacidad de aprender por sí mismo durante toda la vida, en correspondencia con sus aspiraciones, sociales, grupales e individuales y el contexto, en un entorno histórico concreto.

\section{La transformación del nivel de conocimiento didáctico de los docentes de la FACAE}

La falta de dominio de la teoría dela didáctica y los errores en su aplicación práctica conduce a resultados negativos en el proceso de enseñanza-aprendizaje. La Didáctica bien conocida y puesta en práctica conduce a resultados como los descritos en la definición conceptual anterior. Al terminar el curso el $89 \%$ de los docentes de la FACE reconoció el carácter de ciencia de la Didáctica, el 90.7\% aceptó que tiene un objeto de estudio propio, el $92.5 \%$ que posee un sistema teórico que la identifica: Para el 98.1\% dominar el contenido de la Didáctica tiene un nivel de prioridad alto, el $94.4 \%$ planteó que la Didáctica tiene una gran importancia para el desarrollo de sus clases y el 92.45 consideró su dominio de la Didáctica como ciencia entre los niveles alto y medio.

Las definiciones anteriores coinciden en que la Didáctica es una ciencia relativamente joven, en pleno desarrollo, se ubica dentro del segmento de las ciencias de la educación, está relacionada con la Pedagogía y con otras ciencias, tiene una estructura teórica, un objeto de estudio y una función teórica y práctica de marcada vigencia. Estas definiciones son diversas, heterogéneas, dispersas, en algunos casos imprecisas y no permiten la verdadera revelación de la representación gráfica y holística de la Didáctica como ciencia al servicio de los sujetos personalizados de la educación actual. El alcance de estas definiciones cubre a los docentes y excluye a los destinatarios, a los estudiantes para quienes se desarrolla el PEA, lo que evidencia la necesidad de una nueva definición que se acerque más al realidad, que sea más práctica, y valiosa por tanto, y que ofrezca una visón más comprensible para los docentes y más útil para los estudiantes.

La Didáctica es una ciencia que sustenta esta condición en un sistema teórico, todavía imperfecto, en desarrollo ascendente, pero propio, en el que se integran conceptos, definiciones, categorías, leyes, principios y un objeto de estudio que no le corresponde otra ciencia, polémico y posible de perfeccionar, con una esencia propia y particular, cuyo desarrollo está asociado a la investigación y a las experiencias prácticas contextualizadas alrededor de todo lo que sucede en el aula, en función del aprendizaje desarrollador y la formación integral de los estudiantes.

\section{CONCLUSIONES}

De los resultados que se lograron, se derivan las siguientes conclusiones: 1) el estudio permitió determinar como causas fundamentales de la falta de dominio de la Didáctica entre los docentes de la FACAE, la diversidad de enfoques en las definiciones de la Didáctica y la ausencia de capacitación relacionada con esta ciencia, 2) la metodología empleada permitió un estudio integral y crítico de la epistemología de la Didáctica y una definición más integral y contextualizada de la misma, 3) el interés por la Didáctica entre los docentes de la FACAE experimentó un cambio significativo y la valoración de esta ciencia alcanzó un sitial de mayor rango en el modo de actuación de los mismos.

\section{REFERENCIAS}

Abreu, O., Naranjo, M.E., Rhea B., Gallegos, M., Modelo Didáctico para la Facultad de Ciencias Administrativas y Económicas de la Univ. Técnica del Norte de Ecuador, Form. Univ. 9 (4), 3-10 (2016)

Addine, F. y otros 5 autores, Didáctica y optimización del proceso de enseñanza-aprendizaje, Instituto Pedagógico Latinoamericano y Caribeño, 1-54, La Habana, Cuba (1998)

Addine, F. y otros 9 autores, Didáctica Teoría y Práctica., 1ํa Edición, Pueblo y Educación, 1-291, La Habana, Cuba (2004)

Aebli, H., Una didáctica fundada en la psicología de Jean Piaget, Kapelusz, 1-208, Buenos Aires, Argentina (1958) 
Álvarez, C.M., La escuela en la vida, didáctica, Pueblo y Educación, 1-145, La Habana, Cuba (1993)

Álvarez, R.M., Hacia un curriculum integral y contextualizado, Académica, 1-138, La Habana, Cuba (1997)

Alves, L., Compendio de Didáctica General, Kapeluz, 1-412, Buenos Aires, Argentina (1962)

Benedito, V., Introducción a la didáctica, Barcanova, 1-238, Barcelona, España (1987)

Blankertz, H., Didáctica. Conceptos Fundamentales de Pedagogía, 3ª Edición, Herder, 130- 189, Barcelona, España (1981)

Buyse, R., La experimentación pedagógica, Labor, 1-433, Barcelona, España (1964)

Camilloni, M. C., y otros, La formación docente en cuestión: política y pedagógica, Paidós, 1-141, Buenos Aires, Argentina (1994)

Carvajal, M., La didáctica en la Educación, Fundación Academia de Dibujo Profesional, 1-12 (1990)

Comenio, J.A., Didáctica Magna, 8ª Edición, Porrúa, 1-133, Ciudad México, México (1998)

Contreras, J., Enseñanza, currículum y profesorado Introducción crítica a la didáctica, $1^{\text {a }}$ Edición, Akal S.A, 1-245, Madrid, España, 1-245 (1990)

De la Torre, S., Didáctica y currículo bases y componentes del proceso formativo, Dykinson, 1-297, Madrid, España (1993)

Díaz, H., La didáctica universitaria: una alternativa para transformar la enseñanza, Acción Pedagógica, Mérida, 10 (1 y 2), 64-72 (2001)

Díaz, D., La didáctica universitaria: Referencia imprescindible para la enseñanza de calidad, dialnet.unirioja.es/servlet/articulo?código=2795011, Revista Interuniversitaria de formación del profesorado ISSN 1575- 0965 (en línea) 2(1), 1-10 (1999)

Estoker, K., Principios de Didáctica Moderna, Kapelusz, 1-326, Buenos Aires, Argentina (1964)

Estebaranz, A., Didáctica e innovación curricular, $2^{\underline{a}}$ Edición, Secretariado de publicaciones, Universidad de Sevilla, 1-598, Sevilla, España (1999)

Fernández, J., «Didáctica», en Diccionario de Pedagogía, Labor, 1-367, Barcelona, España (1970)

Fernández, A. y Sarramona, J., La educación constante y problemática actual, 2ª Edición, CEAC, 1- 573, Barcelona, España (1984)

Fuentes, H., Didáctica de la educación superior, INPAHU, 1-256, Bogotá, Colombia (2000)

Gervilla, E., Valores de la educación integral, Revista Bordón: Revista de Orientación Pedagógica, ISSN: 0210-5934, 52 (4), https://goo.gl/E6q9JM, 523-536 (2000)

Gottler, J., Pedagogía sistemática, Herder, 1-464, Barcelona, España (1962)

Hashimoto, E., ¿Leyes pedagógicas o leyes didácticas?, UMBRAL, Revista de Educación, Cultura y Sociedad, FACHSE, UNPRG, 6 (11), (en línea), 76-86 (2006)

Herbart, J. F., Pedagogía general derivada del fin de la educación, Espasa- Calpe, 1-267, Madrid, España (1935)

Madrid, D., Mayorga, M.J., ¿Didáctica General en y para Educación Social? Puntos de encuentro desde la perspectiva del alumnado, Revista Educación Siglo XXI, ISSN 1989-466X, http://revistas.um.es/educatio, 28 (2) $245-260$ (2010)

Mallart, J., Didáctica. Concepto, objeto y finalidades. Didáctica General para Psicopedagogos, UNED, 2357, Madrid, España (2001)

Medina, A. y otros 8 autores, Didáctica general, Pearson Educación, 1-480, Madrid, España (2009)

Moreno, T., Didáctica de la Educación Superior: nuevos desafíos en el siglo XXI, Revista Perspectiva Educacional, ISSN: 0718-9729, 50 (2), 26-54 (2011) 
Nérici, I., Hacia una Didáctica General Dinámica, Kapelusz, 1-181, Buenos Aires, Argentina (1973)

Pacios, A., Introducción a la Didáctica, Cincel-Kapelusz, 1-176, Madrid, España (1982)

Pérez, A., Investigación en el aula y paradigma ecológico. Ponencia en el "I Simposio Internacional de Didáctica General y Didácticas Especiales". Murcia, España, 27 de Septiembre al 2 de Octubre (1982)

Pérez, A., Paradigmas contemporáneo de investigación didáctica. Cuadernos de estudio $\mathrm{N}^{\circ} 29$, Laboratorio Educativo, Caracas, Venezuela (1985)

Pérez, A., Sacristán, G., Comprender y transformar la enseñanza, Morata, 1-442, Madrid, España (1992)

Pla, R. y otros 16 autores, Una concepción de la Pedagogía como ciencia desde un enfoque histórico cultural, Pueblo y Educación, 1-79, La Habana, Cuba (2010)

Real, A. Diccionario de la lengua española (23를. Ed.), L.U. ESPASA LIBROS, 1-2432, Madrid, España (2014)

Rosales, C., Didáctica, Núcleos fundamentales, Narcea, 1-24 Madrid, España (1988)

Rosselló, M.R., Didáctica General versus didácticas específicas: un viaje de ida y vuelta, ISSN 2386-7272, Revista Educación y Cultura, 18,133-142 (2005)

Sacristán, J.G., Pérez, A., La enseñanza: su teoría y su práctica, Akal, 1-467, Madrid, España (1989)

Sánchez, M.R., Pedagogía y Didáctica. A Propósito de la Resolución 5436 de 2010. Educación y Ciencia, $15,63-72(2012)$

Sevillano, M. L., Didáctica en el siglo XXI: ejes en el aprendizaje y enseñanza de calidad, McGraw Hill/Interamericana de España, 1-400, Madrid, España (2005)

Stocker. K., Principios de Didáctica moderna, Kapelusz, 1-326, Buenos Aires, Argentina (1966)

Sousa, B., La Universidad en el siglo XXI Para una reforma democrática y emancipatoria de la universidad, 3를 Edición, CIDES-UMSA, ASDI y Plural editores, 1-118, La Paz, Bolivia (2007)

Titone, R., Metodología didáctica, Editorial: RIALP, 1-670, Madrid, España (1970)

Tomachewski, K., Didáctica General, Grijalbo, 1-296, Ciudad de México, México (1970)

Torres, H. y Girón, D.A., Didáctica General. Coordinación Educativa y Cultural Centroamericana. Colección Pedagógica Formación Inicial de Docentes Centroamericanos de Educación Primaria o Básica, 9,121-139 (2009)

Vasco, C. E., algunas reflexiones sobre la pedagogía y la didáctica, 1-10 (1990)

Villalpando, J. M., Manual de Didáctica, Porrúa, 1-192, Ciudad de México, México (1970)

Zabalza, M.A., La didáctica universitaria: una alternativa para transformar la enseñanza, Bordón ISSN: 0210-5934, 59 (2-3), 489-509 (2007) 